\title{
Consistent Modeling of Rotational Nonequilibrium in a Hybrid Particle-Continuum Method
}

\author{
Ashley M. Verhoff* and Iain D. Boyd ${ }^{\dagger}$ \\ Department of Aerospace Engineering, University of Michigan, Ann Arbor, MI 48109
}

\begin{abstract}
Hybrid particle-continuum methods are of interest to the simulation of partially rarefied, hypersonic flows because of the compromise they offer between physical accuracy and numerical efficiency. There are two considerations that are of principal importance to the development of such methods. First, sub-regions of the simulation domain must be assigned to either the particle or continuum solver based on some measure of continuum breakdown. Then, information must be exchanged between these domains in a consistent manner. The present study is concerned with the detection of rotational energy nonequilibrium, and the consistent treatment of rotational-translational energy exchange in both the continuum and particle methods. As such, a new continuum breakdown parameter is proposed and evaluated in the context of a hypersonic, blunt-body flow, and an existing rotational-translational energy exchange model for use in the particle method is verified to be consistent with the corresponding model in the continuum method for a binary gas mixture.
\end{abstract}

\section{Nomenclature}

$C_{h} \quad$ Heat transfer coefficient

$C_{p} \quad$ Surface pressure coefficient

$C_{\tau} \quad$ Shear stress coefficient

$d \quad$ Molecular diameter, $[\AA]$

$E_{\text {coll }} \quad$ Total collision energy, $[J]$

$E_{r e l} \quad$ Relative translational energy, $[J]$

$K n \quad$ Knudsen number

$K n_{G L L} \quad$ Gradient-length local Knudsen number

$k \quad$ Boltzmann constant, $[J / K]$

$M \quad$ Molecular weight, $[\mathrm{kg} / \mathrm{kmol}]$

$m^{*} \quad$ Reduced particle mass, $[\mathrm{kg}]$

$n$ Number density, $\left[\mathrm{m}^{-3}\right]$

$Q \quad$ Generic macroscopic property

$q \quad$ Surface heat flux, $\left[\frac{J}{m^{2} s}\right]$

$R \quad$ Mixture gas constant, $\left[\frac{\mathrm{J}}{\mathrm{kgK}}\right]$

$T$ Temperature, $[K]$

$T^{*} \quad$ Characteristic temperature, $[K]$

$V \quad$ Velocity, $[\mathrm{m} / \mathrm{s}]$

$Z \quad$ Collision number

$\left(Z_{\text {rot }}\right)_{\infty}$ Limiting rotational collision number

$\epsilon \quad$ Error

$\zeta \quad$ Degrees of freedom

$\lambda \quad$ Mean free path, $[m]$

$\nu \quad$ Collision rate, $\left[s^{-1}\right]$

$\rho \quad$ Mass density, $\left[\mathrm{kg} / \mathrm{m}^{3}\right]$

*Graduate Student, Student Member AIAA, averhoff@umich.edu.

†Professor, Fellow AIAA, iainboyd@umich.edu. 


$\begin{array}{ll}\tau_{c} & \text { Mean collision time, }[s] \\ \tau & \text { Relaxation time, }[s], \text { or shear stress, }\left[N / m^{2}\right] \\ \phi & \text { Energy exchange probability } \\ \omega & \text { Variable Hard Sphere (VHS) temperature exponent } \\ \text { Subscripts } \\ p & \text { Species } p \\ q & \text { Species } q \\ p q & \text { Collision class } p q \\ r e f & \text { VHS reference parameters } \\ \text { rot } & \text { Rotational energy mode } \\ \text { trans } & \text { Translational energy mode } \\ \infty & \text { Freestream value }\end{array}$

\section{Introduction}

$\mathrm{T}$ HE finite rate processes that can occur in rarefied, hypersonic flows, such as thermal relaxation and chemical reactions, become important due to the small residence times of the fluid relative to a vehicle of interest. After passing through the strong shock wave caused by a hypersonic vehicle, the kinetic energy once possessed by the gas is converted into random thermal energy, leading to high post-shock temperatures, activation of internal energy modes, and reactions among constituent species of the gas. Each of these processes plays an important role in the design of hypersonic vehicles, and accurate simulation of their coupled nature further complicates the solution procedure.

In the case of blunt-body, hypersonic flows at transitional Knudsen numbers, there exist localized regions of the flowfield, namely, the strong bow shock wave, boundary and shear layers, and the low-density wake, where gradients of macroscopic fluid properties occur over length scales of the same order as the local mean free path. In such regions, there is an insufficient number of collisions among gas molecules/atoms, and equilibrium probability distribution functions of the velocities and/or internal energy states of these particles cannot be maintained. Accurate simulation of these thermodynamic nonequilibrium effects requires use of a kinetic theory-based modeling technique, the most popular of which is the direct simulation Monte Carlo (DSMC) method, first proposed by Bird. ${ }^{1}$ Restrictions on both cell size and time step, however, make the DSMC method computationally expensive for high-density flows. Thus, in terms of numerical efficiency, it is advantageous to use the DSMC method only in regions of the flowfield where a molecular analysis of the fluid is required for physical accuracy, and to employ a more economical computational fluid dynamics (CFD) approach in high-density, near-continuum regions of the flowfield. The development of such a hybrid approach, in which the simulation domain is divided into distinct particle and continuum regions where DSMC and CFD are used, and information is exchanged as the simulation evolves, is of current research interest, and is a desirable alternative, in terms of both numerical efficiency and physical accuracy, to either DSMC or CFD on its own.

The particular hybrid approach that is of interest in the present study is the Modular Particle-Continuum (MPC) method, ${ }^{2}$ which has been in development at the University of Michigan over the past several years. Section II gives an overview of this particular hybrid approach. A closer examination of the rotationaltranslational energy exchange models used in the MPC hybrid method is presented in Section III.A, along with simulation results used to verify consistency between the CFD and DSMC modules of the MPC method. In Section III.B, a revised continuum breakdown parameter for quantifying rotational energy nonequilibrium is proposed and compared to a previously employed continuum breakdown parameter in a hybrid simulation of hypersonic, blunt-body flow at a transitional Knudsen number. Finally, conclusions are drawn based on these results, and future extensions of this work are recommended.

\section{Modular Particle-Continuum Method}

The MPC method is a hybrid framework that loosely couples the LeMANS ${ }^{3}$ finite volume CFD solver with the MONACO ${ }^{4}$ DSMC code. LeMANS is capable of numerically solving the steady, laminar NavierStokes equations with second order spatial accuracy, and MONACO is a general cell-based implementation 
of the DSMC method. The independent development and use of LeMANS and MONACO, in addition to the modular nature of the MPC method, allow for this particular hybrid approach to be maintained as state-of-the-art. Both the numerical efficiency and the physical accuracy of the MPC method relative to full DSMC and full CFD have been demonstrated for one-dimensional shock waves, ${ }^{5}$ and two-dimensional and axisymmetric, steady, hypersonic, blunt-body flows. ${ }^{2,6}$ In addition, the MPC method has been parallelized using the message passing interface (MPI) and METIS ${ }^{7}$ graph partitioning, and extended to simulate rotational and vibrational nonequilibrium effects in simple gases. ${ }^{8,9}$

The hybrid procedure is initialized with a converged CFD solution from LeMANS, from which a number of continuum breakdown parameters are calculated and employed to demarcate particle and continuum domains. Boyd, et al. ${ }^{10}$ suggested the use of a local Knudsen number to indicate breakdown of the linear constitutive relationships assumed for closure of the Navier-Stokes equations. Unlike the global Knudsen number, $K n_{\infty}$, which is the ratio of the freestream mean free path, $\lambda_{\infty}$, to a characteristic length scale of a vehicle or phenomenon of interest, the gradient-length local Knudsen number is calculated as

$$
K n_{G L L-Q}=\lambda\left|\frac{\nabla Q}{Q}\right|
$$

where $Q$ is a macroscopic fluid property such as translational temperature, rotational temperature, individual species mass density, or velocity magnitude, and $\lambda$ is the local mean free path. It was also shown in Ref. 10 that a solution obtained with CFD would agree to within $5 \%$ of that obtained using DSMC when the $K n_{G L L-Q}$ had a maximum value below 0.05 . Therefore, cells with values of this continuum breakdown parameter exceeding this threshold are labeled as part of the particle domain. Otherwise, if the $K n_{G L L-Q}$ does not have a value greater than 0.05 , the cell remains part of the continuum domain.

In addition to the gradient-length local Knudsen number, Schwartzentruber, et al. ${ }^{11}$ found that it was necessary to use an additional continuum breakdown parameter specifically indicative of rotational nonequilibrium in the post-shock region of hypersonic, blunt-body flows. This supplementary parameter is given by

$$
K n_{R O T-N E Q}=\frac{1}{2} \frac{T_{\text {trans }}-T_{\text {rot }}}{T_{\text {rot }}},
$$

and is a relative difference between the translational and rotational temperatures, with a coefficient determined by Deschenes, et al. ${ }^{9}$ such that the same threshold value of 0.05 could continue being used. It is one of the objectives of the present work to replace this parameter with a more physically intuitive local Knudsen number calculated as a function of the local mean thermal speed and characteristic relaxation time of the rotational energy mode. More detail on this parameter is given in Section III.B.

Upon determining which regions of the flow domain should be simulated using DSMC in order to achieve physical accuracy, and which regions should be simulated using CFD in order to minimize computational cost, the original mesh is refined to meet the cell size requirements of the DSMC method. The particle domain is then extended a user-defined number of "buffer" cells and populated with DSMC simulator particles consistent with a Chapman-Enskog velocity distribution function (VDF), which describes a small-order perturbation from an equilibrium, or Maxwellian, VDF. An illustration of the hybrid interfaces and their associated terminology is shown in Figure 1.

The hybrid procedure then enters the MONACO DSMC module, where simulator particles are moved and selected for collision as per standard DSMC algorithms, with new particles generated in and exiting particles removed from DSMC boundary cells at every time step. In order to mitigate the statistical scatter associated with DSMC when evaluating macroscopic properties for updating CFD boundary conditions and recalculating continuum breakdown parameters, the subrelaxation average of Sun and Boyd ${ }^{12}$ is used. The particle domain solution continues to evolve as the DSMC procedures of moving and colliding particles are repeated, allowing for the hybrid interfaces to be updated. Only after these hybrid interfaces cease to move is information from the particle domain first transferred to the continuum domain in the form of updated boundary conditions. The MPC procedure then enters the LeMANS CFD module, where the continuum domain solution is advanced. After this cycle is repeated a number of times, and the hybrid interfaces adjust to their final positions, the hybrid interfaces are locked in place. From this point on, the hybrid method alternates between updating the continuum domain and sampling particle data in the DSMC domain until the statistical scatter is sufficiently reduced. 


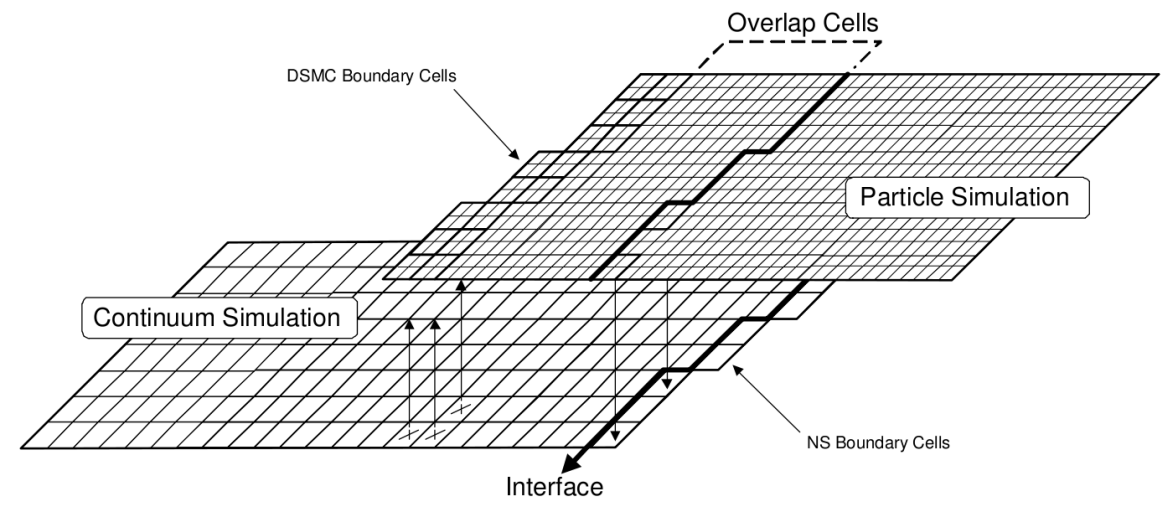

Figure 1. Illustration of hybrid interface. ${ }^{6}$

\section{Rotational Nonequilibrium Effects}

Two aspects of a hybrid particle-continuum method require special consideration as the functionality of the method improves. These are the appropriate placement of the hybrid interfaces in terms of both physical accuracy and numerical efficiency, and the use of consistent physical models in the CFD and DSMC component modules. The work presented here aims to address these concerns as they pertain to the rotational energy mode of a partially rarefied gas mixture.

\section{III.A. Rotational-Translational Energy Exchange}

The use of consistent physical models in DSMC and CFD is of utmost importance to the accurate transfer of information across the hybrid interfaces. Because placement of the hybrid interfaces is limited to regions of near-continuum, where both numerical techniques are physically accurate, consistent physical models ensure consistent information transfer, and guarantee that any differences between the results obtained using these two computational methods are due to differences between the numerical procedures only. As such, one of the objectives of the current work is to determine rotational-translational energy exchange models to be used in DSMC and CFD that yield equivalent results, specifically when simulating a mixture of chemical species. Whereas this topic has been explored in terms of macroscopic temperatures in simple gases by Deschenes, et al., ${ }^{9}$ detailed comparisons of rotational collision numbers were omitted and are, thus, further investigated here.

The CFD module of the MPC hybrid method employs Parker's model, ${ }^{13}$ which has the following form:

$$
Z_{\text {rot }}=\frac{\left(Z_{\text {rot }}\right)_{\infty}}{1+\frac{\pi^{3 / 2}}{2}\left(\frac{T^{*}}{T_{\text {trans }}}\right)^{1 / 2}+\left(\frac{\pi^{2}}{4}+\pi\right) \frac{T^{*}}{T_{\text {trans }}}} .
$$

In Eq. (3), $Z_{r o t}$ is the rotational collision number as defined for continuum methods, while $\left(Z_{r o t}\right)_{\infty}$ and $T^{*}$ are species-specific constants. In the case of $N_{2}$, these constants are assigned values of 18.1 and $91.5 \mathrm{~K}$, respectively. The product of the rotational collision number and the mean collision time for a given species $p$,

$$
\left(\tau_{c}\right)_{p}=\left(\nu_{c}\right)_{p}^{-1}=\left[\sum_{q=1}^{s}\left(\nu_{c}\right)_{p q}\right]^{-1}=\left[\sum_{q=1}^{s} 2 \pi^{1 / 2} d_{r e f, p q}^{2} n_{q}\left(\frac{T_{\text {trans }}}{T_{r e f, p q}}\right)^{1-\omega_{p q}}\left(\frac{2 k T_{r e f, p q}}{m_{p q}^{*}}\right)^{1 / 2}\right]^{-1},
$$

yields the characteristic rotational energy relaxation time for that species. In Eq. (4), $d_{r e f}$ is the reference diameter used in the Variable Hard Sphere (VHS) collision model, ${ }^{1} T_{r e f}$ is the reference temperature, $\omega$ is the temperature exponent in the viscosity-temperature power law relationship, $m_{p q}^{*}$ is the reduced particle mass of the collision class $p q$, and $n$ is the number density. The collision rate, $\nu$, is simply the reciprocal of the collision time for each species.

In order to maintain consistency with the model used in CFD, Boyd's variable probability rotational relaxation model ${ }^{14}$ is used in the DSMC module of the MPC hybrid method. This model was derived such 
that, under equilibrium conditions, the integral over all instantaneous probabilities of rotational-translational energy exchange would yield an average probability that would be consistent with the inverse of the rotational collision number predicted by Parker's model. It should be noted that use of this instantaneous probability in a particle simulation requires a particle-continuum correction factor derived by Lumpkin, et al. ${ }^{15}$ Boyd proposed two different approaches to calculate the probabilities that should be used to determine whether or not rotational-translational energy exchange occurs in each particle collision. The first assumed that the instantaneous probabilities are functions of the relative translational energy of the colliding pair and, thus, involved integration over the collision-biased equilibrium probability distribution function for relative velocity. The final form of the instantaneous probability of rotational-translational energy exchange derived in this way is

$$
\phi_{\text {rot }}=\frac{Z_{\text {trans }}}{\left(Z_{\text {rot }}\right)_{\infty}}\left[1+\frac{\Gamma\left(\frac{1}{2} \zeta\right)}{\Gamma\left(\frac{1}{2} \zeta-\frac{1}{2}\right)}\left(\frac{k T^{*}}{E_{\text {rel }}}\right)^{1 / 2} \frac{\pi^{3 / 2}}{2}+\frac{\Gamma\left(\frac{1}{2} \zeta\right)}{\Gamma\left(\frac{1}{2} \zeta-1\right)} \frac{k T^{*}}{E_{\text {rel }}}\left(\frac{\pi^{2}}{4}+\pi\right)\right],
$$

where $E_{r e l}$ is the relative energy of the colliding particles, and $\zeta$ is the number of degrees of freedom corresponding to the relative energy, as biased by the collision selection process, which is equal to $5-2 \omega$ in this formulation. The translational energy collision number, $Z_{\text {trans }}$, is assigned a value of unity. The drawback of this approach, however, is that equipartition of energy is not inherently maintained because of the preferential acceptance of collision pairs with lower relative translational energies to undergo rotationaltranslational energy exchange. Since this preferential treatment does not include the rotational energy mode, the Borgnakke-Larsen ${ }^{16}$ internal energy exchange model will tend to redistribute more of the energy to the translational mode than is appropriate to maintain equipartition of energy. Thus, the cell-based average of these instantaneous probabilities, calculated for all collision pairs regardless of actual rotational-translational energy exchange, must be used.

This averaging procedure is not necessary if the instantaneous probabilities are assumed to be functions of the total collision energy of the particle pair. Therefore, Boyd's second approach employed the equilibrium probability distribution function for total collision energy in order to obtain an average probability that would be consistent with Parker's model in CFD. The instantaneous probability defined in this way has the same form as Eq. (5) except $E_{r e l}$ is replaced by $E_{\text {coll }}$, i.e. the sum of the relative translational energy of the colliding pair and the rotational energies of both particles, and $\zeta$ is now the sum of the degrees of freedom associated with the rotational and relative energies.

The latter of these DSMC models was successfully used in past MPC simulations of simple gases exhibiting rotational nonequilibrium. ${ }^{9}$ When used in isothermal heat bath simulations of $50 \% \mathrm{~N}_{2} / 50 \% \mathrm{~N}$ (by mole), however, this approach provides DSMC results that compare less favorably with CFD. The rotational temperature profiles from these simulations, in which the translational temperature is held constant at $10,000 \mathrm{~K}$, and the rotational temperature is initialized to $1,000 \mathrm{~K}$, are shown in Figure 2(a). The greatest discrepancy between CFD and DSMC in this case is approximately $213 \mathrm{~K}$, or $3.39 \%$ of the CFD rotational temperature at that time. This is sizable considering the simplicity of the current simulation relative to simulations of interest, which may include vibrational nonequilibrium and chemical reactions. Examination of the rotational collision numbers also shows marked disagreement, as shown in Figure 2(b). It should be noted that the rotational temperatures and collision numbers cannot be made to agree even upon use of various average temperatures in Parker's model so as to incorporate the effects of the rotational energy mode. The particular CFD results shown in Figures 2(a) and 2(b) employ an average temperature based on the degrees of freedom of the relative energy of a particular collision pair, and the total number of rotational energy degrees of freedom of that pair. Therefore, because the degrees of freedom change depending upon the collision class, so does the relevant temperature used in the calculation of the rotational collision number.

In the interest of clarity, the mixture average rotational collision number for $N_{2}$ is calculated as

$$
\left(Z_{r o t}\right)_{N_{2}}=\frac{\left(\tau_{r o t}\right)_{N_{2}}}{\left(\tau_{c}\right)_{N_{2}}}=\left(\tau_{r o t}\right)_{N_{2}}\left(\nu_{N_{2}-N_{2}}+\nu_{N_{2}-N}\right)
$$

where

$$
\left(\tau_{r o t}\right)_{N_{2}}=\left[\frac{\nu_{N_{2}-N_{2}}}{\left(Z_{r o t}\right)_{N_{2}-N_{2}}}+\frac{\nu_{N_{2}-N}}{\left(Z_{r o t}\right)_{N_{2}-N}}\right]^{-1}
$$


In the above equations, $\left(\tau_{r o t}\right)_{N_{2}}$ is the characteristic rotational relaxation time of $N_{2}$, which is a result of $N_{2}$ molecules undergoing relaxation events with $N$ atoms and other $N_{2}$ molecules. The rotational collision number for each collision class is considered separately because they are slightly different when calculated as a function of a relevant average temperature rather than the translational temperature only.

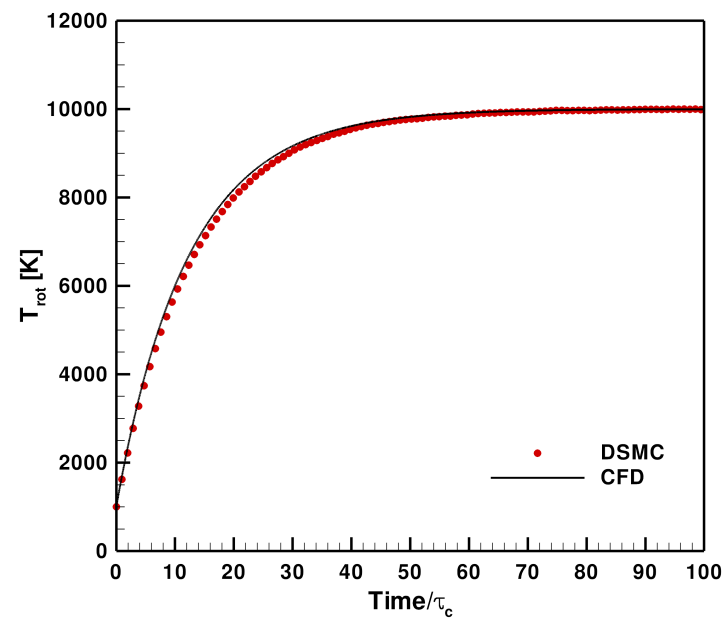

(a) Rotational temperature

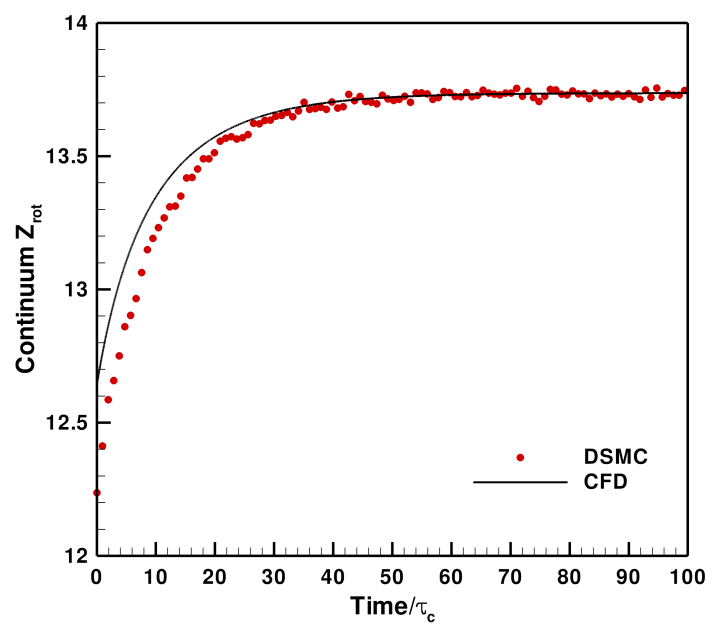

(b) Rotational collision number

Figure 2. Isothermal heat bath results, with $\phi_{\text {rot }}\left(E_{\text {coll }}\right)$.

When the rotational-translational energy exchange probability is assumed to be a function of the relative translational energy only, the agreement between CFD and DSMC is improved. For the same isothermal heat bath, the resulting rotational temperature profiles are shown in Figure 3(a), where the maximum discrepancy has been reduced to approximately $37 \mathrm{~K}$, which is $0.74 \%$ of the CFD rotational temperature at that time. The rotational collision numbers also appear to be in much better agreement, as shown in Figure 3(b). In addition, because the rotational collision number is constant throughout the simulation since this is an isothermal heat bath, the rotational temperature profiles are also compared to the theoretical relaxation profile in Figure 3(a), the form of which was proposed by Jeans. ${ }^{17}$

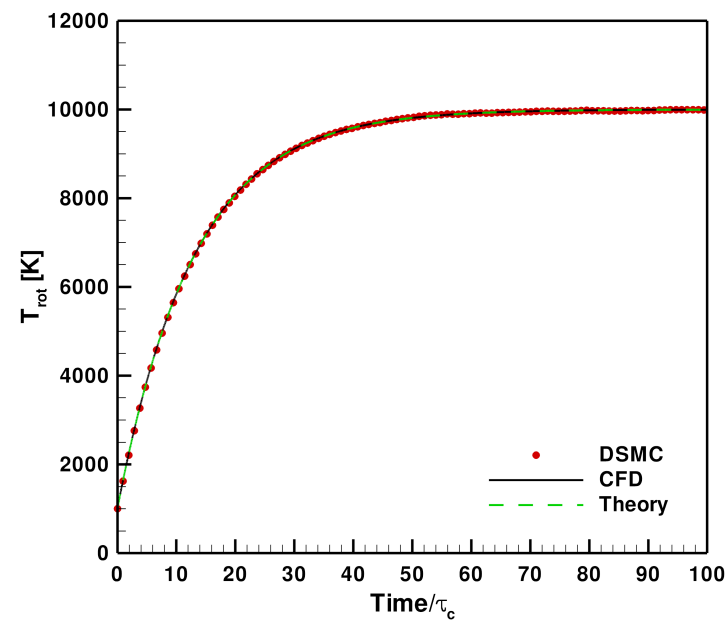

(a) Rotational temperature

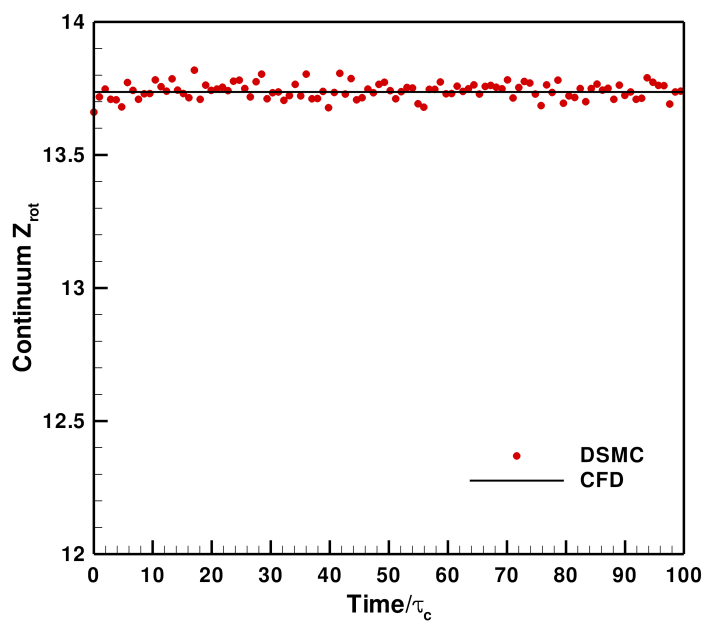

(b) Rotational collision number

Figure 3. Isothermal heat bath results, with $\phi_{\text {rot }}\left(E_{r e l}\right)$. 
As previously mentioned, various averaging procedures were used to incorporate the effect of the rotational energy mode in Parker's model for CFD, and the results showing the most favorable agreement with DSMC have been shown in Figures 2(a) and 2(b). None were found to be capable of replicating the complex relationship between the total collision energy and rotational collision number exhibited by Boyd's model in which the energy exchange probability is assumed to be a function of the total collision energy. Therefore, all subsequent hybrid simulations in which rotational nonequilibrium effects are present will employ Boyd's model in particle domains, where the probability of rotational-translational energy exchange is assumed to be a function of the relative translational energy only. Such a model will provide better agreement with CFD at the hybrid interfaces in terms of the macroscopic rotational temperature and rotational collision number, and will alleviate concerns about inconsistency between the rotational-translational energy exchange models. This is important not only in its own right, but also when moving forward to flows with more complex physics in the future.

\section{III.B. Continuum Breakdown Detection}

Accurate determination of continuum breakdown is the mechanism for achieving the required balance between physical accuracy and numerical efficiency that is the underlying goal of the MPC hybrid method. If hybrid interfaces are not located in regions of near-continuum, the CFD module will be used beyond its range of applicability and physical accuracy will deteriorate. In contrast, being too conservative in the placement of hybrid interfaces leads to excessive use of the DSMC method in regions that can be accurately simulated using a more economical continuum approach. As mentioned earlier, in addition to the gradient-length local Knudsen number, previous MPC simulations have employed a continuum breakdown parameter dependent on the relative difference between translational and rotational temperatures. Although this parameter is proportional to the rotational-translational energy relaxation source term in the rotational energy equation used in CFD, it is desirable to develop a parameter for detecting rotational energy nonequilibrium that is similar to the gradient-length local Knudsen number and would, therefore, have more of a physical significance. The new parameter that is proposed and evaluated in this work is

$$
K n_{\text {rot }}=\lambda_{\text {rot }}\left|\frac{\nabla T_{\text {rot }}}{T_{\text {rot }}}\right|=\left(\tau_{\text {rot }}\right) \sqrt{\frac{8 R T_{\text {trans }}}{\pi}}\left|\frac{\nabla T_{\text {rot }}}{T_{\text {rot }}}\right|=\left(Z_{\text {rot }}\right) \lambda\left|\frac{\nabla T_{\text {rot }}}{T_{\text {rot }}}\right|,
$$

which is the same as the gradient-length local Knudsen number evaluated as a function of $T_{\text {rot }}$ except for the presence of the rotational collision number. This is not the same as simply using a different threshold value for $K n_{G L L}\left(T_{r o t}\right)$ because the rotational collision number is a function of the translational temperature. The parameter in Eq. (8) attempts to incorporate in the gradient-length local Knudsen number the fact that more collisions are required for equilibration of the rotational energy mode than for the translational mode. In the interest of thoroughness, the efficacy of the current continuum breakdown parameters should also be evaluated periodically as the capabilities of the MPC hybrid method continue to be developed. As such, this new parameter is evaluated alongside those currently used to detect continuum breakdown, and the results of this analysis are presented here. Because the rotational energy nonequilibrium parameter given in Eq. (2) was originally employed to extend the post-shock hybrid interface further downstream, the bow shock region is the focus of the following analysis.

The flowfield of interest is that of a Mach 10 flow of a $50 \% N_{2} / 50 \% N$ (by mole) gas mixture over a two-dimensional cylinder at a global Knudsen number of 0.01. The freestream temperature is specified to be $217 \mathrm{~K}$, the freestream velocity is $3,590 \mathrm{~m} / \mathrm{s}$, and the cylinder surface employs an isothermal boundary condition where the temperature is maintained at 1,000 K. Full CFD, full DSMC, and hybrid results obtained using the MPC method are presented and discussed. The full CFD solution is verified to be grid-independent, and this grid is also used in the MPC method, where the particle regions of the grid are automatically refined in order to comply with DSMC cell size requirements. In order to obtain consistent measures of computational cost, the full DSMC simulation employs the same grid that was refined by and for use in the hybrid method. Both the full CFD simulation and the CFD module of the MPC method employ a point-implicit time integration method, where a maximum CFL number of 10 is used. A constant time step of $1 \cdot 10^{-8} s$ is used in both the full DSMC simulation and the DSMC module of the MPC method; this time step is verified to be less than the local mean collision time throughout the flow domain. In order to maintain a minimum of 40 particles per cell (20 particles of each species per cell), a constant numerical 
weight of $2 \cdot 10^{12}$ is specified, except for the wake region of the cylinder, where low densities require the use of a numerical weight of $4 \cdot 10^{10}$.

The models used to simulate rotational nonequilibrium effects in both CFD and DSMC have been discussed extensively in the previous section. The relaxation times used in the vibrational energy equation of the continuum formulation are determined from curve-fits obtained by Millikan and White. ${ }^{18}$ By employing a particle-continuum correction factor, ${ }^{19}$ Deschenes, et al. ${ }^{8}$ showed that this same model could be used in DSMC to obtain vibrational-translational energy exchange results that are consistent with CFD. Transport properties of the mixture are calculated using Gupta's Mixing Rule ${ }^{20}$ and the VHS collision model ${ }^{1}$ in CFD and DSMC, respectively. The VHS collision model parameters used in this work are given in Ref. 21, along with a discussion on how they were obtained and verified to be consistent with collision integral data from Wright, et. al. ${ }^{22}$

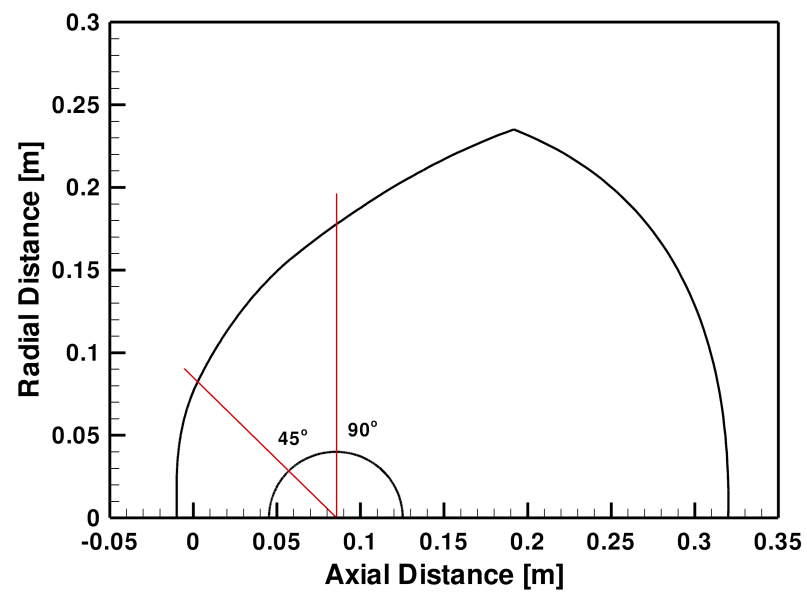

Figure 4. Computational domain and data extraction lines.

The results from full CFD and full DSMC simulations are compared along several extraction lines, which are illustrated in Figure 4. A comparison of the full CFD and full DSMC simulation results along the stagnation streamline is shown in Figure 5. The error, $\epsilon$, which is plotted against the distance from the cylinder surface, normalized by the cylinder radius, is defined as

$$
\epsilon=\frac{Q_{C F D}}{Q_{D S M C}}-1
$$

Two dashed black lines are also included here to indicate the maximum acceptable error of CFD relative to DSMC, whether an underestimate or overestimate. Remember that the continuum breakdown parameter threshold value of 0.05 has been associated with a maximum allowable difference of $5 \%$ between full DSMC and full CFD simulation results. In Figure 5, it is shown that the difference between the translational temperatures yielded by full CFD and full DSMC is the first flow property to exceed $5 \%$ in the pre-shock region. This occurs at a normalized distance of 0.801 from the cylinder surface. The macroscopic parameter that defines the initial position of the post-shock hybrid interface is the rotational temperature, where an error of $5 \%$ last occurs at a normalized distance of 0.382 . These regions where CFD and DSMC differ by more than $5 \%$ can be compared to the values of various continuum breakdown parameters in order to assess their effectiveness. The continuum breakdown parameters that have been previously mentioned are calculated as a function of the full CFD solution, and their profiles along the stagnation streamline are shown in Figure 6. It will be shown that the pre-shock hybrid interface moves upstream by a relatively large distance as the hybrid simulation of this flowfield proceeds, and therefore, the adequacy of the continuum breakdown parameters in this region cannot be assessed from full CFD results. However, the post-shock hybrid interface location experiences minimal change throughout the course of a hybrid simulation. From Figure 6, it can be seen that the $K n_{R O T-N E Q}$ parameter exceeds the threshold value of 0.05 , which is indicated by the dashed black line, at a normalized distance of 0.373 , and that the $K n_{G L L}\left(T_{\text {rot }}\right)$ parameter does so at a distance of 0.422 . The former would stipulate a post-shock hybrid interface that is conservative, whereas the latter would place the post-shock hybrid interface too far upstream. 


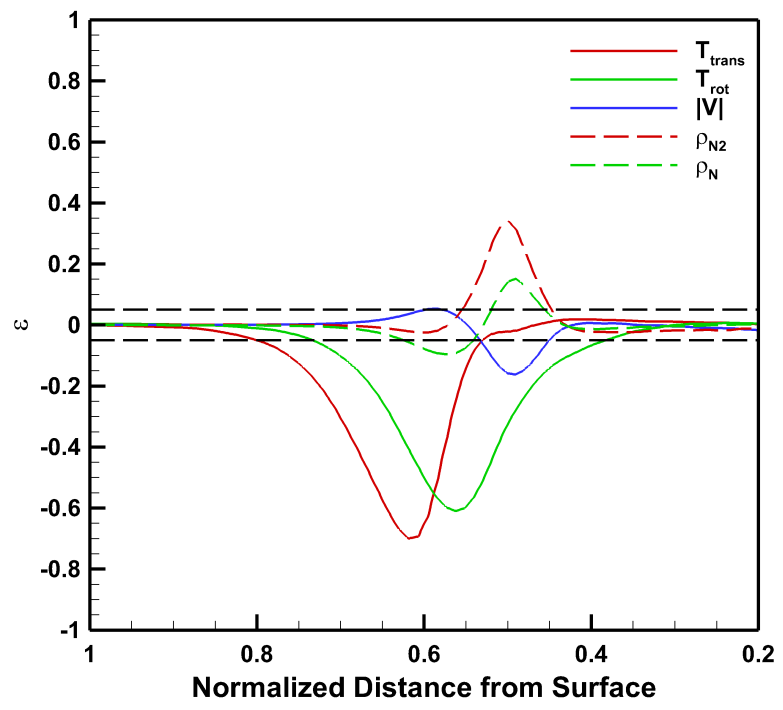

Figure 5. Errors between full CFD and full DSMC results along stagnation streamline.

If the new $K n_{\text {rot }}$ parameter is now considered, it can be seen that at a normalized distance of 0.375 , which is slightly less conservative than that given by $K n_{R O T-N E Q}$, this parameter has a value of 0.2 . Because an appropriate threshold value has not yet been determined for the new continuum breakdown parameter, a tentative value of 0.2 could be assumed, and the new $K n_{\text {rot }}$ parameter would yield results that are comparable to those of the original $K n_{R O T-N E Q}$ parameter.

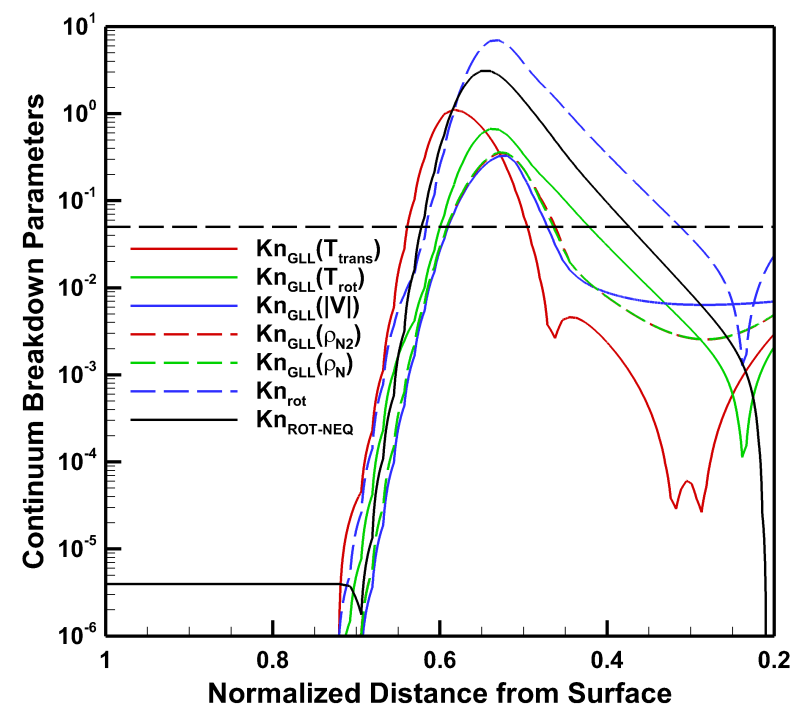

Figure 6. Continuum breakdown parameters based on full CFD solution along stagnation streamline.

Similar comparisons can be made for results along extraction lines at $45^{\circ}$ and $90^{\circ}$. Figure 7 shows a plot of the error in macroscopic properties between full CFD and full DSMC along the $45^{\circ}$ extraction line, and Figure 8 shows the corresponding profiles of the continuum breakdown parameters of interest in this paper. Again, the difference between the rotational temperatures obtained with CFD and DSMC is the last to decrease below 5\% in the post-shock region. As illustrated in Figure 7, this occurs at a normalized distance of 0.550 . From Figure 8, it can be seen that $K n_{R O T-N E Q}$ dictates that the post-shock hybrid interface should be located at a normalized distance of 0.545 , which is downstream of where the error in the 
rotational temperature decreases below $5 \%$, and $K n_{G L L}\left(T_{\text {rot }}\right)$ predicts that the interface should be located at a normalized distance of 0.594 , which would still be in a region of nonequilibrium. If a threshold value of 0.2 is again used for the $K n_{\text {rot }}$ parameter, then this continuum breakdown parameter indicates that the hybrid interface should be placed at a normalized distance of 0.541 . This prediction is slightly more conservative than that of the $K n_{R O T-N E Q}$ parameter.

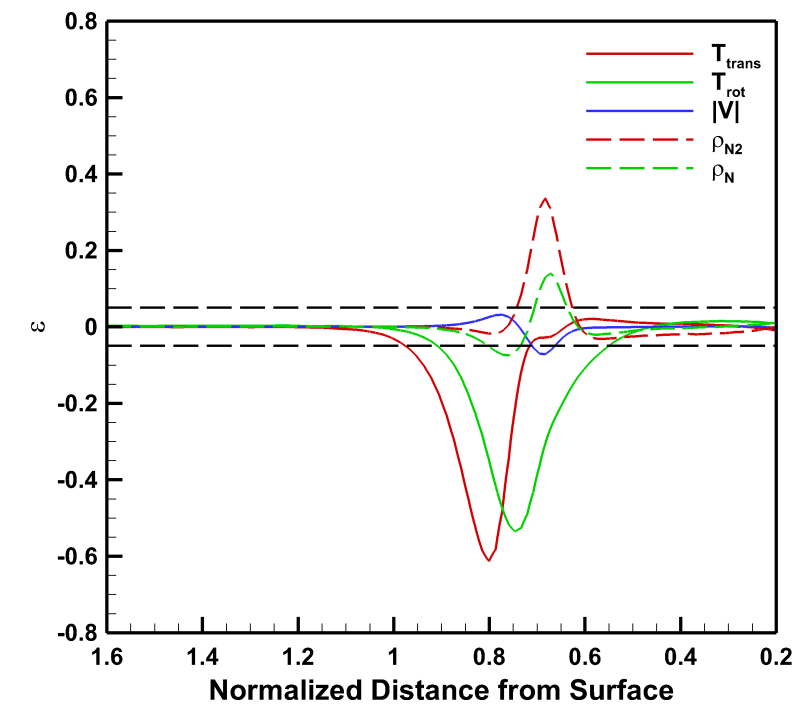

Figure 7. Errors between full CFD and full DSMC results along $45^{\circ}$ extraction line.

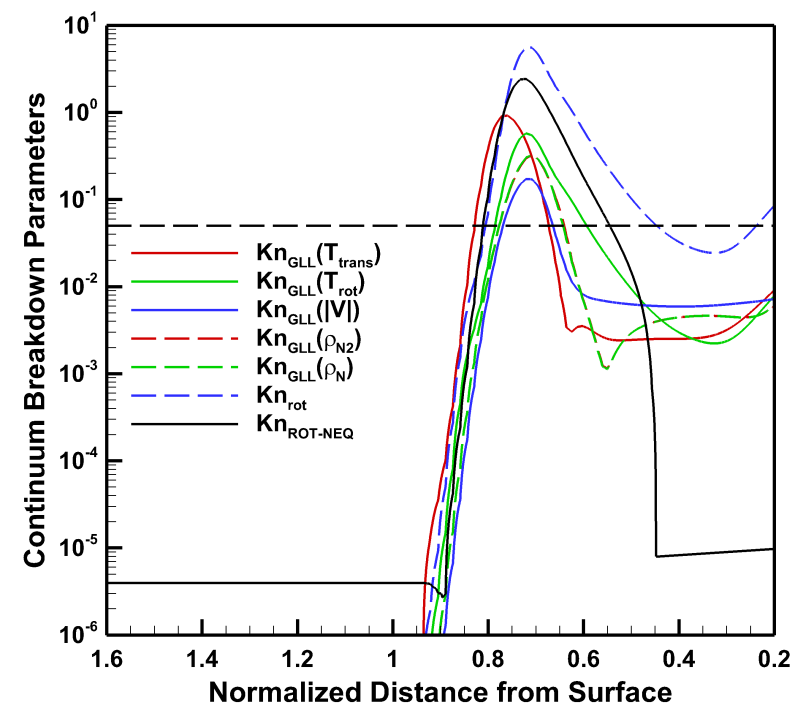

Figure 8. Continuum breakdown parameters based on full CFD solution along $45^{\circ}$ extraction line.

A final comparison of macroscopic properties obtained using full CFD and full DSMC along the $90^{\circ}$ extraction line is shown in Figure 9. As was the case with the stagnation streamline and the $45^{\circ}$ extraction line, the difference between the CFD and DSMC results for rotational temperature stipulates the initial location of the post-shock hybrid interface, and that it should be placed at a normalized distance of 1.502 . Both the $K n_{R O T-N E Q}$ and $K n_{G L L}\left(T_{\text {rot }}\right)$ parameters, the profiles of which are shown in Figure 10, fail to predict post-shock interface locations that are this distance away from or closer to the cylinder surface. The interface locations indicated by these parameters are a normalized distance of 1.503 and 1.571 away from 
the cylinder, respectively, placing the interface in a nonequilibrium region of the flowfield. In contrast, the new $K n_{\text {rot }}$ parameter predicts that continuum breakdown occurs at a normalized distance of 1.494, which is appropriate based on the errors in macroscopic properties shown in Figure 9. A threshold value of 0.2 is again used for this new parameter.

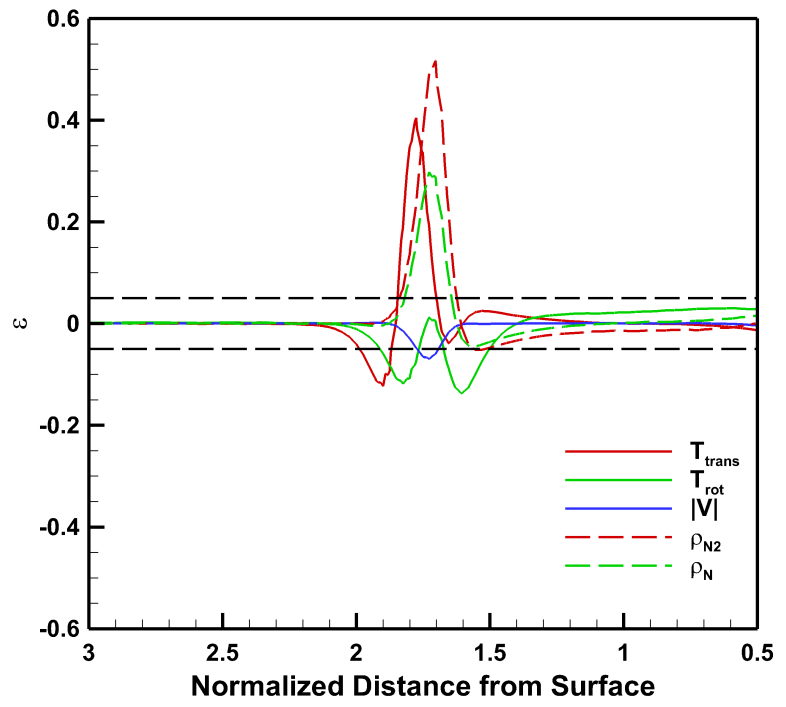

Figure 9. Errors between full CFD and full DSMC results along $90^{\circ}$ extraction line.

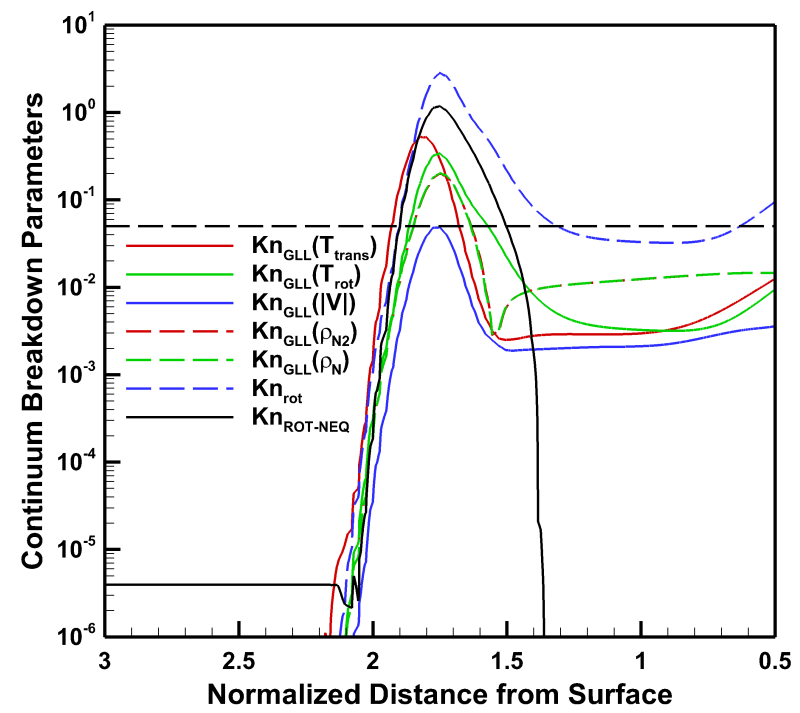

Figure 10. Continuum breakdown parameters based on full CFD solution along $90^{\circ}$ extraction line.

The continuum breakdown parameter results presented here are based on a full CFD solution only, which is known to be incorrect in specific regions of hypersonic flowfields. Therefore, it is not sufficient to assess these parameters and their ability to predict continuum breakdown based on these results alone; it is necessary to continue the evaluation throughout the course of a hybrid simulation. Because the MPC hybrid method is initialized with a full CFD solution, the analysis presented above is an evaluation of the initial placement of the hybrid interfaces, and is not an accurate assessment of the overall effectiveness of the continuum breakdown parameters that are used. For completeness, the locations of the final hybrid interfaces 
are evaluated by comparing the full DSMC results to the hybrid results at the point in the simulation where they are locked in place. Note that the final hybrid interfaces are determined and then standard DSMC sampling commences, so that the interfaces are adjusted based on the subrelaxation average used in the transient portion of the hybrid simulation. In addition, there is a smoothing algorithm employed to mitigate the occurrence of isolated regions of either particle or continuum domains, so the interface locations indicated by the employed continuum breakdown parameters are approximate.

After several hybrid cycles, during which information is exchanged between the particle and continuum domains of the flowfield, and the solution in each of these domains is advanced, the hybrid interfaces have settled to their final positions and are locked in place. Depending on the continuum breakdown parameters that are used, the relative sizes of the CFD and DSMC domains and the locations of the hybrid interfaces may differ. It is one of the objectives of the current study to assess the new $K n_{\text {rot }}$ parameter relative to the original $K n_{R O T-N E Q}$ parameter. Comparisons of the full DSMC results and hybrid results along the stagnation streamline at the time when the hybrid interfaces are locked are shown in Figures 11(a) and 11(b). In the former, the $K n_{R O T-N E Q}$ parameter is used along with the gradient-length local Knudsen numbers based on translational temperature, rotational temperature, velocity magnitude, and individual species mass density, whereas the original parameter is replaced with the new $K n_{\text {rot }}$ parameter in the latter figure. As is evident in Figure 11(a), use of the original $K n_{R O T-N E Q}$ parameter fails to obtain hybrid particle-continuum results that differ from full DSMC results by less than $5 \%$. At some point along the stagnation streamline, all of the macroscopic properties examined here exhibit errors in excess of $5 \%$. However, the hybrid results obtained by using the new $K n_{\text {rot }}$ parameter are in excellent agreement with full DSMC, as shown in Figure 11(b).

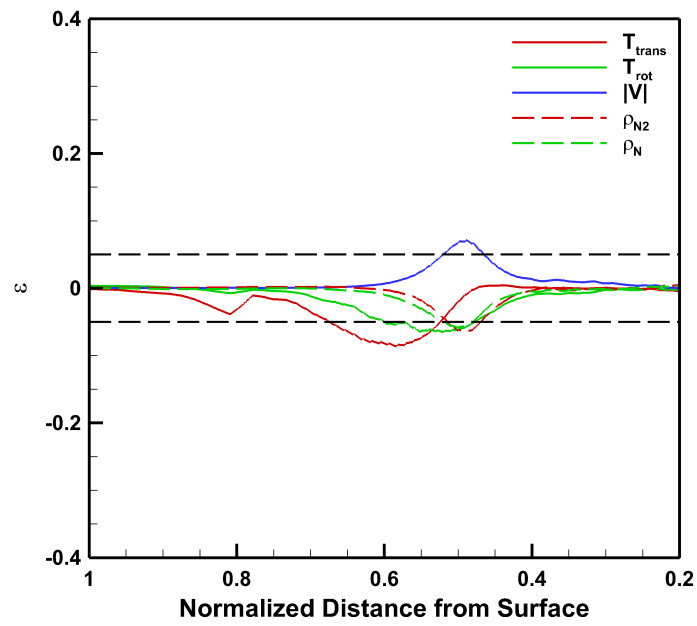

(a) $K n_{R O T-N E Q}$

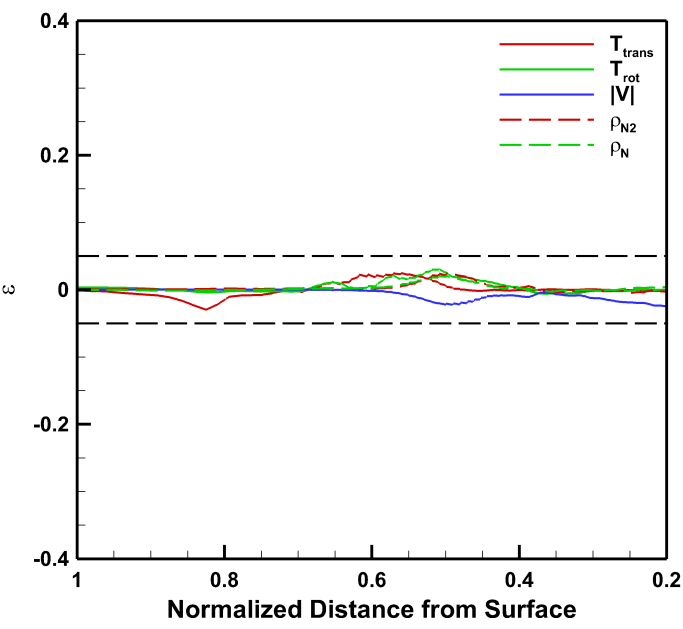

(b) $K n_{\text {rot }}$

Figure 11. Errors between hybrid and full DSMC results along stagnation streamline.

Similarly, the hybrid results along a $45^{\circ}$ extraction line are compared with those from a full DSMC solution. The results in which the original $K n_{R O T-N E Q}$ parameter is employed for the hybrid simulation are plotted in Figure 12(a) and those in which the new $K n_{\text {rot }}$ parameter is used are shown in Figure 12(b). Again, the hybrid results obtained by using the new $K n_{\text {rot }}$ parameter differ by less than $5 \%$ relative to the full DSMC results, whereas differences in translational temperature, rotational temperature, $N_{2}$ mass density, and $N$ mass density exceed this requirement for maximum error when the original parameter is used.

Finally, comparisons between hybrid results obtained with the original $K n_{R O T-N E Q}$ parameter and full DSMC, and comparisons between hybrid results obtained with the new $K n_{\text {rot }}$ parameter and full DSMC along a $90^{\circ}$ extraction line are plotted in Figure 13(a) and Figure 13(b), respectively. Neither set of continuum breakdown parameters enables the MPC hybrid code to produce results accurate to within $5 \%$ of full DSMC. There is a noticeable reduction in the errors associated with the macroscopic properties of interest when the new $K n_{\text {rot }}$ parameter is used, however. The maximum error, whether $K n_{R O T-N E Q}$ or $K n_{\text {rot }}$ is used, occurs in the translational temperature, and this error is reduced from a value of -0.226 to -0.127 when the original parameter is replaced with the new continuum breakdown parameter. 


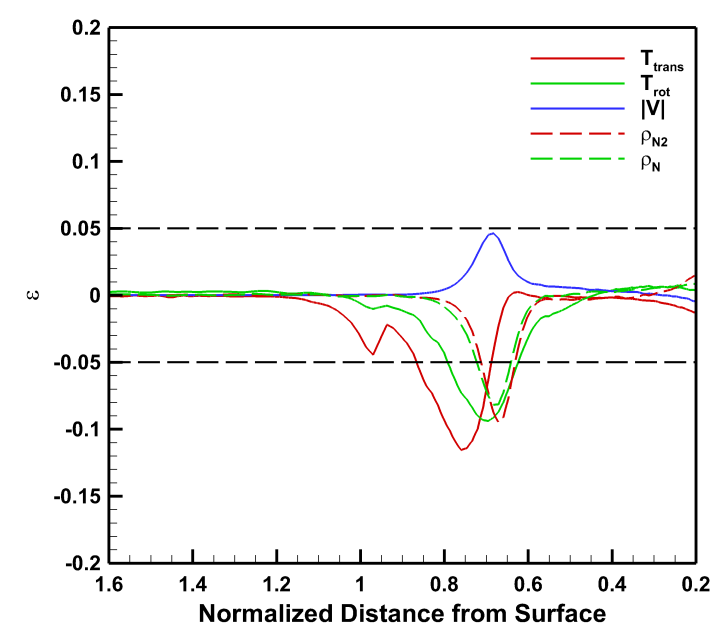

(a) $K n_{R O T-N E Q}$

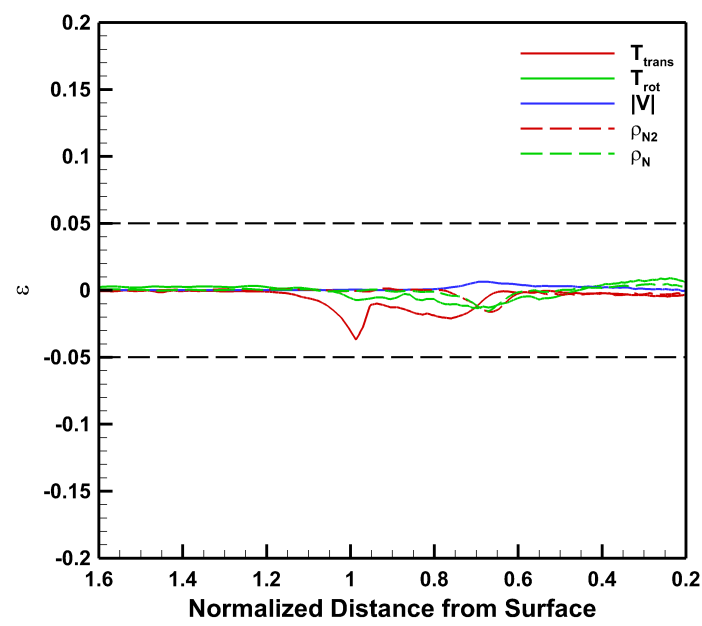

(b) $K n_{\text {rot }}$

Figure 12. Errors between hybrid and full DSMC results along $45^{\circ}$ extraction line.

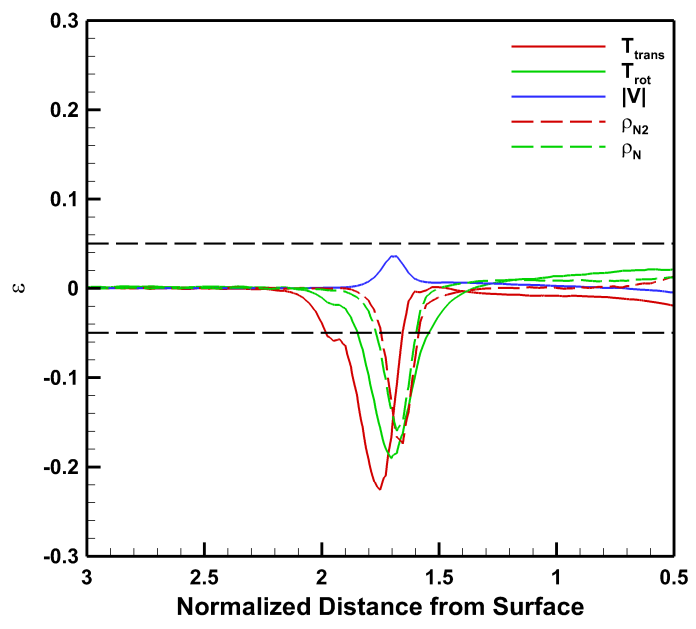

(a) $K n_{R O T-N E Q}$

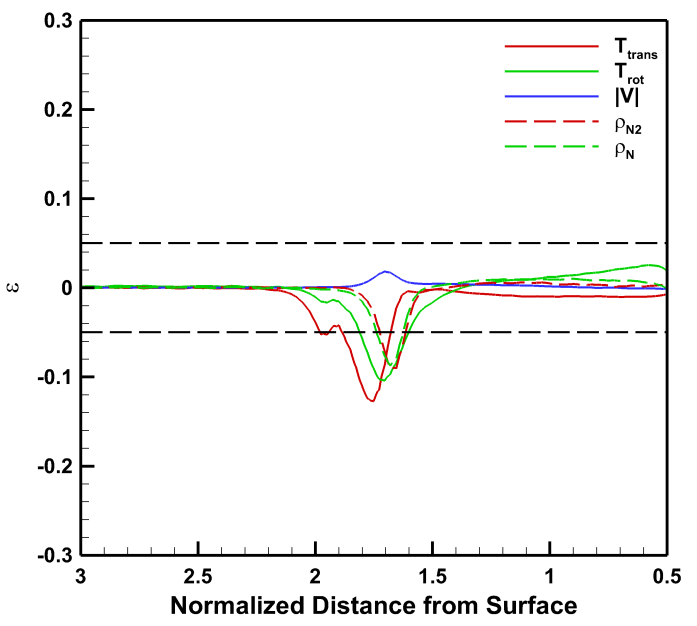

(b) $K n_{\text {rot }}$

Figure 13. Errors between hybrid and full DSMC results along $90^{\circ}$ extraction line.

Looking now at the flowfield as a whole, the initial and final hybrid interfaces as determined using either the original $K n_{R O T-N E Q}$ parameter or the new $K n_{\text {rot }}$ parameter, along with the gradient-length local Knudsen numbers based on translational temperature, rotational temperature, individual species mass density, and velocity magnitude, are shown in Figure 14. As previously mentioned, the initial position of the post-shock hybrid interface is a good approximation of its final location. There appears to be little difference between the two sets of results in terms of the size and shape of the particle region surrounding the shock. The particle domain encompassing the boundary layer and wake region, however, is very different depending on which rotational nonequilibrium parameter is used. The new $K n_{\text {rot }}$ parameter forces a markedly larger particle region in the forebody as compared to $K n_{R O T-N E Q}$. Accurately simulating the flowfield in this region is of heightened importance because the flow is subsonic and therefore able to influence the upstream solution. In addition, the $K n_{\text {rot }}$ parameter also indicates a larger particle domain surrounding the wake region. 


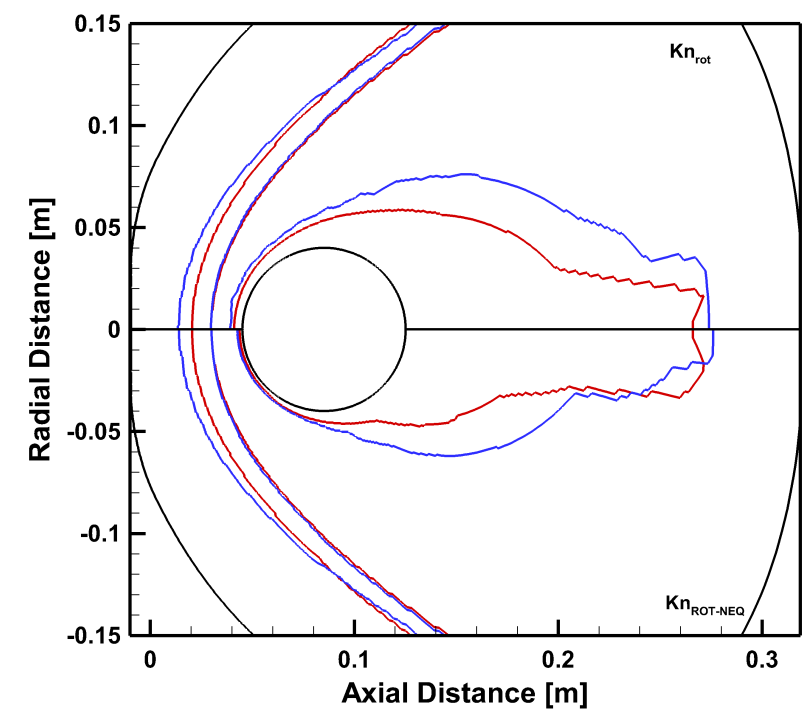

Figure 14. Initial (red) and final (blue) hybrid interfaces, where $K n_{\text {rot }}$ is used in the upper pane, and $K n_{R O T-N E Q}$ is used in the lower pane.

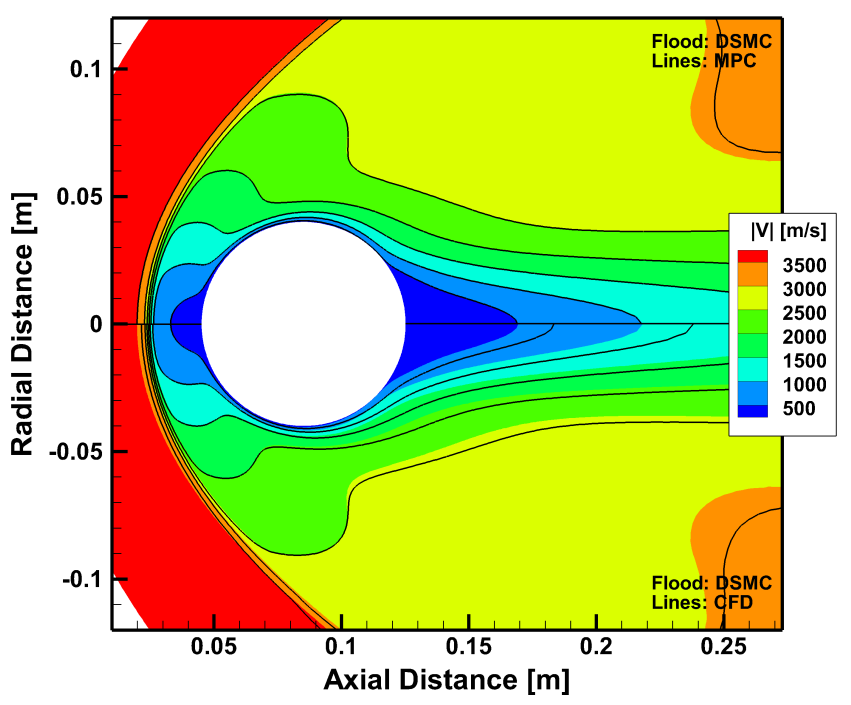

Figure 15. Contours of velocity magnitude.

Somewhat surprisingly, there is very little difference between the two hybrid solutions in terms of flowfield properties. Therefore, only those contours obtained with the new rotational nonequilibrium parameter are compared to those obtained with full DSMC and full CFD in the following figures. Figure 15 shows contours of velocity magnitude throughout the simulation domain, with the flooded contours representing the solution from full DSMC, the black lines in the upper pane representing the MPC hybrid results, and the black lines in the lower pane representing the full CFD results. This same presentation scheme is used for all the flowfield results shown here. The hybrid results are nearly indistinguishable from those obtained with full DSMC except for the far wake region. As expected, full CFD predicts a thinner shock and a delayed shock onset relative to the full DSMC and hybrid results. Full CFD also appears to over predict the size of the wake region because of the no-slip boundary condition employed at the cylinder surface, whereas both full DSMC and the particle region delineated at the surface in the hybrid method allow for velocity slip. Similar 
conclusions can be drawn from Figures 16, 17, and 18, which show contours of translational, rotational, and vibrational temperatures, respectively. Differences between the contours of vibrational temperature predicted by full DSMC and the MPC hybrid method appear to be the most noticeable, although this is misleading to some extent because the range of temperatures covered by this energy mode is much smaller than for the translational and rotational energy modes.

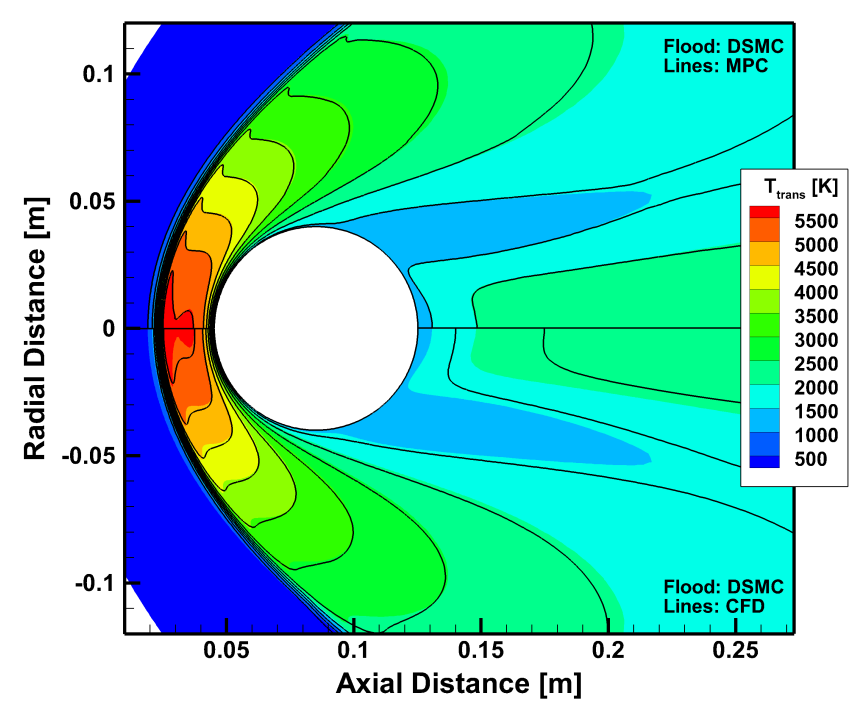

Figure 16. Contours of translational temperature.

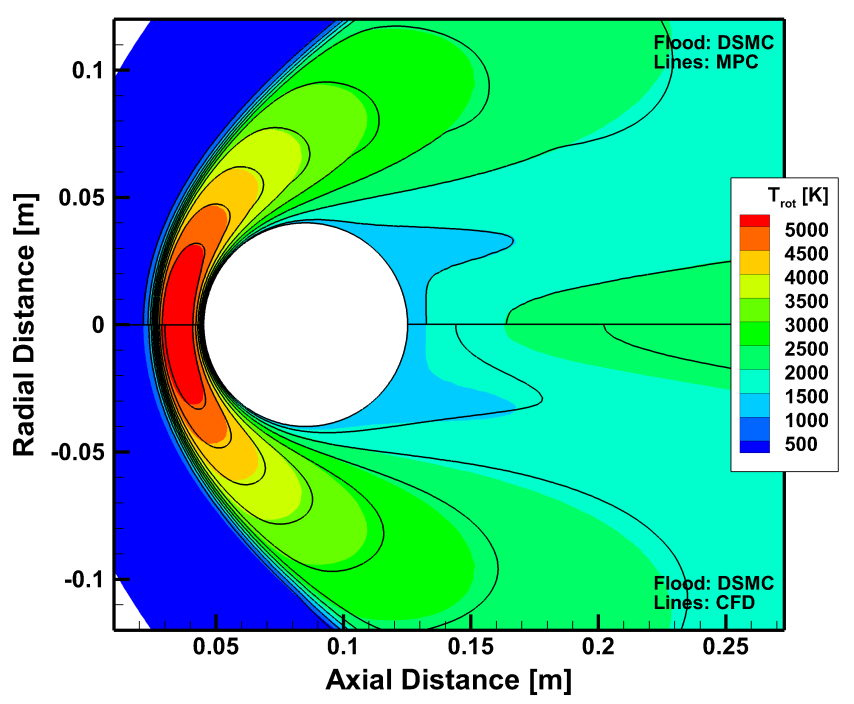

Figure 17. Contours of rotational temperature.

There do exist subtle differences between the two hybrid solutions, as previously shown along the stagnation streamline and $45^{\circ}$ and $90^{\circ}$ extraction lines. These differences are also evident in the cylinder surface properties, specifically the heat transfer coefficient, where hybrid results obtained using the $K n_{R O T-N E Q}$ parameter and those from full DSMC disagree by as much as 10\%, as shown in Figure 19. The surface property coefficients calculated in this paper are defined as follows, where $C_{h}$ is the heat transfer coefficient, $C_{p}$ is the surface pressure coefficient, and $C_{\tau}$ is the shear stress coefficient: 


$$
\begin{aligned}
C_{h} & =\frac{q_{\infty}}{\frac{1}{2} \rho_{\infty} V_{\infty}^{3}}, \\
C_{p} & =\frac{p}{\frac{1}{2} \rho_{\infty} V_{\infty}^{2}}, \\
C_{\tau} & =\frac{|\tau|}{\frac{1}{2} \rho_{\infty} V_{\infty}^{2}} .
\end{aligned}
$$

It is suspected that the thickness of the DSMC region near the cylinder surface is under estimated by the original $K n_{R O T-N E Q}$ parameter, and that this incorrect placement of the hybrid interface leads to an under prediction in the heat flux coefficient along the entire cylinder surface. The heat flux coefficient profile predicted by the hybrid solution in which the new $K n_{\text {rot }}$ parameter is used, however, is in excellent agreement with the full DSMC result; the maximum difference between these two profiles is less than $3 \%$. As expected, the heat flux coefficient profile predicted by full CFD is everywhere greater than that given by full DSMC. The continuum approach forces the temperature near the wall to match the isothermal condition at the wall, which leads to higher temperature gradients and, in turn, larger heat fluxes. Differences between the two hybrid solutions are less noticeable in the profiles of surface pressure coefficient along the cylinder surface, which is plotted in Figure 20. Here, the hybrid solution obtained by employing the original $K n_{R O T-N E Q}$ parameter under predicts the pressure coefficient along the cylinder surface by as much as $4 \%$, whereas the hybrid solution obtained by using the new parameter agrees to within $2 \%$ of the full DSMC solution.

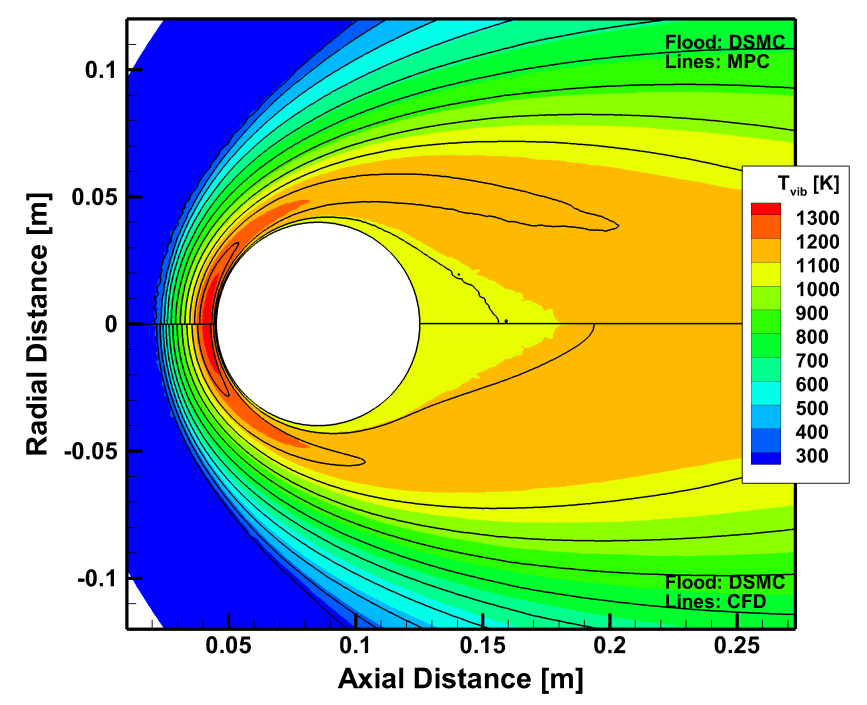

Figure 18. Contours of vibrational temperature.

Profiles of the shear stress coefficient obtained using full DSMC, full CFD, and the MPC hybrid method are shown in Figure 21. Hybrid results obtained using the original $K n_{R O T-N E Q}$ parameter disagree with full DSMC results by as much as 7\%; this error is reduced to approximately $4 \%$ when the new rotational nonequilibrium parameter is used. Due to the no-slip condition enforced by CFD, the continuum prediction of the shear stress coefficient exceeds those of the full DSMC and hybrid simulations, starting at an angular position of approximately $30^{\circ}$. The size of the wake region is also larger in the continuum solution, as is evident by the sudden change in slope of the shear stress coefficient, which is based on the absolute value of wall shear stress.

It should also be mentioned that the computational cost of using the new $K n_{\text {rot }}$ parameter is comparable to that of using the original rotational nonequilibrium parameter. In the former solution, approximately 38 million particles are used in the DSMC domains of the hybrid simulation, which is a remarkable reduction compared to the 82 million particles that are required in the full DSMC simulation. Use of the original $K n_{R O T-N E Q}$ parameter requires approximately 34 million particles, which is due to the decreased size of 
particle regions defined in the boundary layer and wake in this simulation. Even when the new parameter is used, however, an MPC hybrid solution is obtained with only half of the CPU time required by a full DSMC simulation.

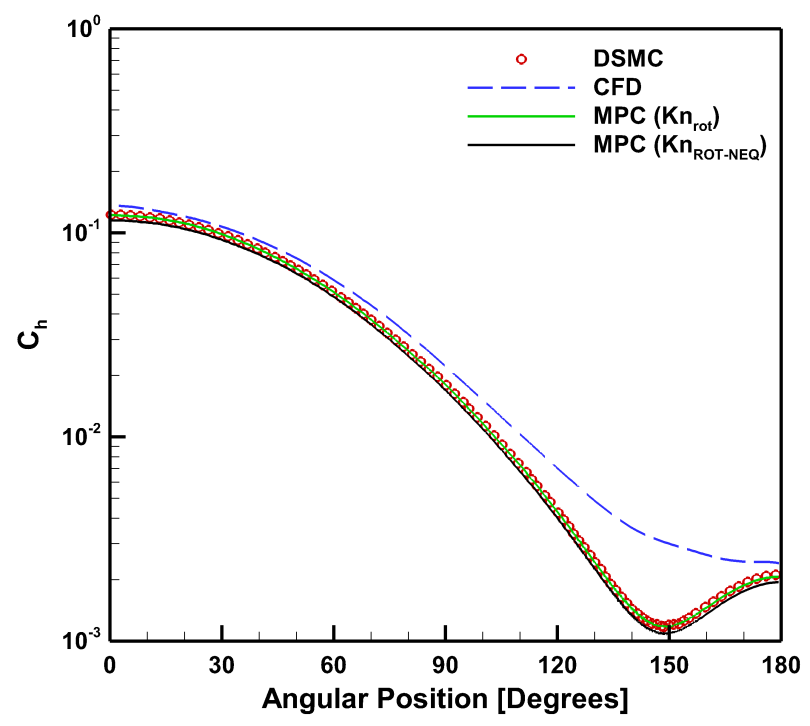

Figure 19. Heat transfer coefficient along cylinder surface.

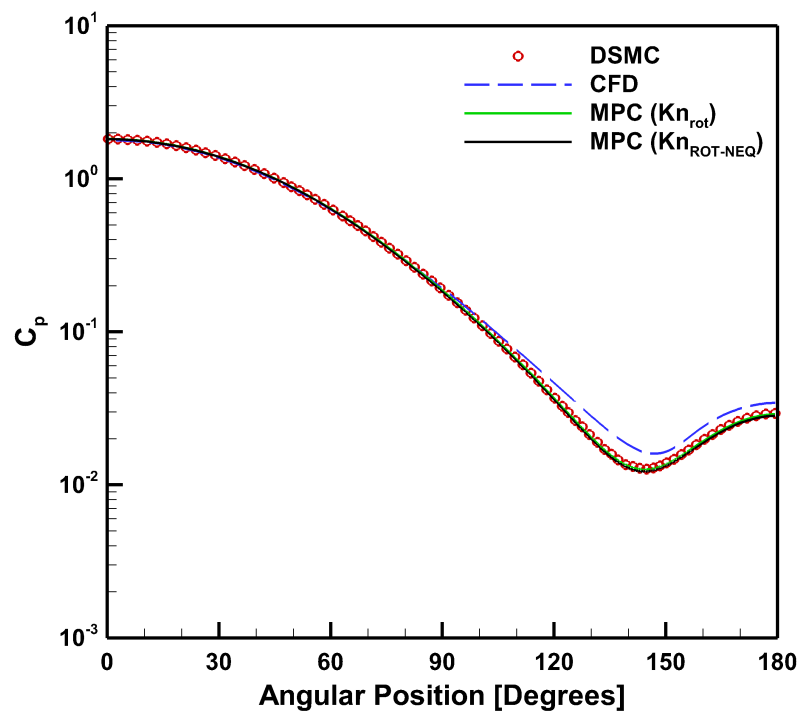

Figure 20. Surface pressure coefficient along cylinder surface.

\section{Conclusions and Future Work}

The two main concerns when developing a hybrid particle-continuum method are the appropriate placement of hybrid interfaces, and the consistent and accurate exchange of information between continuum and particle domains. In order to ensure that the rotational-translational energy exchange model used in the DSMC module of the MPC hybrid method is consistent with that used in the CFD module, simulations of isothermal heat baths were performed and the transient relaxation profiles of rotational temperature and 


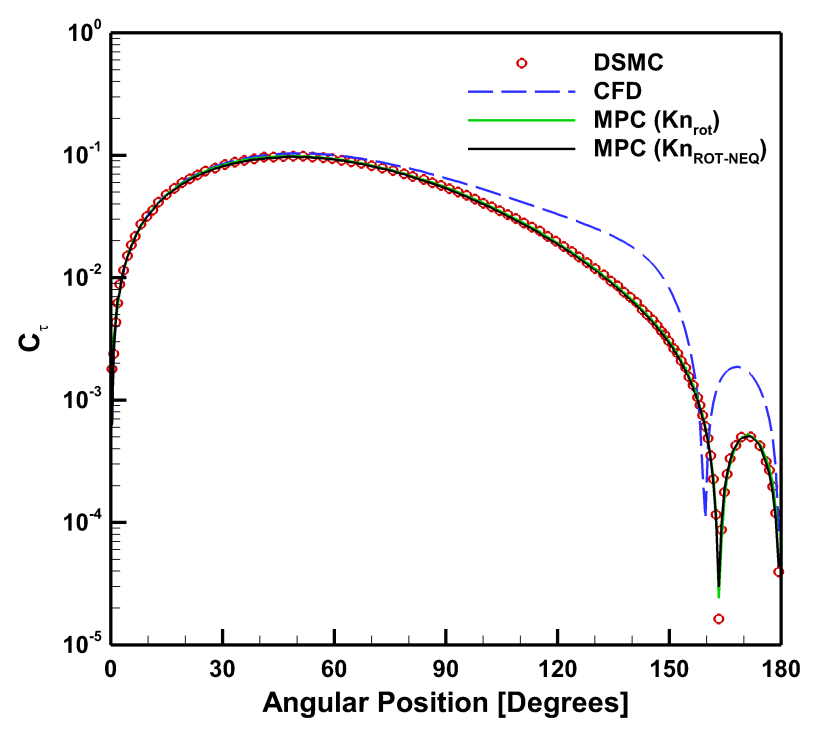

Figure 21. Shear stress coefficient along cylinder surface.

rotational collision number were compared. It was determined that consistency was obtained when the instantaneous probability of rotational-translational energy exchange in DSMC is assumed to be a function of the relative translational energy only. This is due to the continuum model for calculating the rotational collision number being a function of translational energy. Attempting to replicate the complex relationship between total collision energy and the energy exchange probability exhibited in DSMC proved unsuccessful, even when an effective temperature was used in the continuum model.

In addition, a new continuum breakdown parameter for detecting rotational nonequilibrium was proposed and compared to a parameter that was previously employed in MPC hybrid simulations. This new parameter is physically intuitive because it has the form of a local Knudsen number, and its use in the MPC hybrid method yielded improved accuracy when compared to full DSMC simulation results. This was illustrated through comparisons of surface properties and macroscopic fluid properties along various extraction lines, although the differences in many of the flowfield results were not notable. Because the rotational nonequilibrium parameter was originally devised so as to extend the post-shock hybrid interface downstream, this research effort focused on the particle domain surrounding the shock. However, it was shown that the newly proposed parameter also increased the thickness of the particle domain in the boundary layer, which may have contributed to the more accurate hybrid results obtained with this parameter relative to the original. Other hybrid interfaces, specifically the interface in the boundary layer and wake region, will be the focus of future investigation. Depending on the outcome of that analysis, other continuum breakdown parameters may also be proposed and evaluated, such as a gradient-length local Knudsen number based on species mass fractions, which would be indicative of the diffusion effects present in a gas mixture.

\section{Acknowledgments}

This research was conducted with government support under and awarded by the Department of Defense, Air Force Office of Scientific Research, National Defense Science and Engineering Graduate (NDSEG) Fellowship, 32 CFR 168a. The use of computational resources made available through the NASA Advanced Supercomputing Division is also an integral part of this research, and is greatly appreciated.

\section{References}

\footnotetext{
${ }^{1}$ Bird, G. A., Molecular Gas Dynamics and the Direct Simulation of Gas Flows, Oxford University Press, 1994.

${ }^{2}$ Schwartzentruber, T. E., Scalabrin, L. C., and Boyd, I. D., "A modular particle-continuum numerical method for hypersonic non-equilibrium gas flows," J. Comput. Phys., Vol. 225, No. 1, July 2007, pp. 1159-1174.
} 
${ }^{3}$ Martin, A., Scalabrin, L. C., and Boyd, I. D., "High performance modeling of atmospheric re-entry vehicles," J. Phys.: Conf. Ser., Vol. 341, No. 012002, 2012.

${ }^{4}$ Dietrich, S. and Boyd, I. D., "Scalar and parallel optimized implementation of the direct simulation Monte Carlo method," J. Comput. Phys., Vol. 126, No. 2, July 1996, pp. 328-342.

${ }^{5}$ Schwartzentruber, T. E. and Boyd, I. D., "A hybrid particle-continuum method applied to shock waves," J. Comput. Phys., Vol. 215, No. 2, July 2006, pp. 402-416.

${ }^{6}$ Schwartzentruber, T. E., Scalabrin, L. C., and Boyd, I. D., "Multiscale particle-continuum simulations of hypersonic flow over a planetary probe," J. Spacecraft Rockets, Vol. 45, No. 6, November-December 2008, pp. 1196-1206.

${ }^{7}$ Karypis, G. and Kumar, V., "METIS: A software package for partitioning unstructured graphs, partitioning meshes, and computing fill-reducing orderings of sparse matrices," University of Minnesota, MN, 1998.

${ }^{8}$ Deschenes, T. R. and Boyd, I. D., "Extension of a modular particle-continuum method to vibrationally excited, hypersonic flows," AIAA J., Vol. 49, No. 9, September 2011.

${ }^{9}$ Deschenes, T. R., Holman, T. D., and Boyd, I. D., "Effects of rotational energy relaxation in a modular particle-continuum method," J. Thermophys. Heat Tr., Vol. 25, No. 2, April-June 2011.

${ }^{10}$ Boyd, I. D., Chen, G., and Candler, G. V., "Predicting failure of the continuum fluid equations in transitional hypersonic flows," Phys. Fluids, Vol. 7, No. 1, January 1995, pp. 210-219.

${ }^{11}$ Schwartzentruber, T. E., Scalabrin, L. C., and Boyd, I. D., "Hybrid particle-continuum simulations of nonequilibrium hypersonic blunt-body flowfields," J. Thermophys. Heat Tr., Vol. 22, No. 1, January-March 2008.

${ }^{12}$ Sun, Q. and Boyd, I. D., "Evaluation of macroscopic properties in the direct simulation Monte Carlo method," J. Thermophys. Heat Tr., Vol. 19, No. 3, July-September 2005, pp. 329-335.

${ }^{13}$ Parker, J. G., "Rotational and vibrational relaxation in diatomic gases," Phys. Fluids, Vol. 2, No. 4, July-August 1959, pp. 449-462.

${ }^{14}$ Boyd, I. D., "Analysis of rotational nonequilibrium in standing shock waves of nitrogen," AIAA J., Vol. 28, No. 11, 1990, pp. 1997-1999.

${ }^{15}$ Lumpkin, F. E., Haas, B. L., and Boyd, I. D., "Resolution of differences between collision number definitions in particle and continuum simulations," Phys. Fluids, Vol. 3, No. 9, 1991, pp. 2282-2284.

${ }^{16}$ Borgnakke, C. and Larsen, P. S., "Statistical collision model for Monte Carlo simulation of polyatomic gas mixutre," J. Comput. Phys., Vol. 18, No. 4, August 1975, pp. 405-420.

${ }^{17}$ Jeans, J. H., The Dynamical Theory of Cases, Cambridge University Press, 1916.

${ }^{18}$ Millikan, R. C. and White, D. R., "Systematics of vibrational relaxation," J. Chem. Phys., Vol. 39, No. 12, December 1963, pp. 3209-3213.

${ }^{19}$ Gimelshein, N. E., Gimelshein, S. F., and Levin, D. A., "Vibrational relaxation rates in the direct simulation Monte Carlo method," Phys. Fluids, Vol. 14, No. 12, December 2002, pp. 4452-4455.

${ }^{20}$ Gupta, R. N., Yos, J. M., Thompson, R. A., and Lee, K. P., "A review of reaction rates and thermodynamic and transport properties for an 11-species air model for chemical and thermal nonequilibrium caclulations to 30,000 K," NASA-RP-1232, 1990.

${ }^{21}$ Verhoff, A. M. and Boyd, I. D., "Extension of a hybrid particle-continuum method for a mixture of chemical species," 28th International Symposium on Rarefied Gas Dynamics, AIP Conf. Proc. 1501, 2012, pp. 342-349.

${ }^{22}$ Wright, M. J., Bose, D., Palmer, G. E., and Levin, E., "Recommended collision integrals for transport property computations, Part 1: Air species," AIAA J., Vol. 43, No. 12, December 2005, pp. 2558-2564. 